\title{
NUEVO MÉTODO DE ESTERIUZACIÓN AMBULATORIA Y PERMANENTE CON TÉCNICA HISTEROSCÓPICA, ESSURE
}

\author{
Vicente Solá D. ${ }^{1}$, Jack Pardo S. ${ }^{1}$, Paolo Ricci A. ${ }^{1}$, Enrique Guiloff F. ${ }^{1}$ \\ ${ }^{1}$ Unidad de Ginecología, Departamento de Ginecología y Obstetricia, Clínica Las Condes.
}

\section{RESUMEN}

Objetivo: Presentamos nuestra experiencia en la inserción de un nuevo método de esterilización permanente vía histeroscópica, Essure. Se revisa el procedimiento de inserción, complicaciones, tolerancia, grado de satisfacción y aceptación de usuaria. Método: Entre enero y febrero de 2005 se realizaron 5 esterilizaciones con Essure en la Unidad de Ginecología y Obstetricia de Clínica Las Condes. Resultados: Se colocó el dispositivo en ambas trompas exitosamente en todas las pacientes. Rango de tiempo operatorio: 10-30 minutos. No hubo complicaciones intraoperatorias. Todas expresaron buena tolerancia y un alto grado de satisfacción frente al procedimiento de inserción histeroscópico. Lo recomendarían por su rapidez, carácter ambulatorio y ausencia de dolor. Durante el postoperatorio un caso presentó metrorragia leve y algia pélvica moderada. Conclusiones: Nuestra primera experiencia en la inserción del dispositivo intra tubario mostró ser un procedimiento bien tolerado y con buena aceptación por las pacientes.

\section{PALABRAS CLAVES: Esterilización permanente, dispositivo intra tubario, histeroscopía, esterilización ambulatoria}

\section{SUMMARY}

Objective: We presented our experience in Essure insertion, a new hysteroscopic permanent sterilization method. We reviewed insertion procedure, complications, degree of tolerance and acceptance by user. Method: Between January and February 2005 five sterilizations cases with Essure were made in Las Condes Clinic. Results: The device in both tubes in all women was placed. Operative time was between 10 and 30 minutes. Intraoperative complications did not appear. All patients expressed very good tolerance and a high degree of satisfaction with insertion procedure. The reason because they would recommend it was: rapidity, ambulatory and absence of pain. During postoperative time one case presented weighs uterus bleeding and moderate pain. Conclusion: Our first experience in hysteroscopic intra Fallopian tube device insertion showed a procedure with very good tolerance and acceptance by the patients.

\section{KEY WORDS: Permanent sterilization, intra Fallopian tube device, hysteroscopy, ambulatory sterilization}

\section{INTRODUCCIÓN}

La esterilización tubaria ha sido el método de anticoncepción permanente más utilizado en el mundo (1,2). Aprovechando las bondades de la cirugía mínimamente invasiva, actualmente se rea- liza mayoritariamente por vía laparoscópica $(3,4)$. Sin embargo, al igual que la esterilización tubaria efectuada por minilaparatomía abdominal, se debe exponer a la paciente a incisiones abdominales y anestesia, las cuales no están exentas del riesgo de complicaciones $(5,6)$. 
A principios de los años 70 con el desarrollo de la histeroscopia, se comienzan a desarrollar métodos de esterilización por esta vía (7). Entre estos se encuentran los métodos de oclusión tubaria por diferentes dispositivos como la silicona $(8,9)$. Sin embargo, estos métodos oclusivos ofrecían obstrucciones incompletas y tasas altas de expulsión con falla del método (10).

En 1996 se desarrolla un nuevo dispositivo para esterilización permanente por histeroscopía. Se trata de un espiral que produce una obstrucción tubaria por medio de fibrosis; inicialmente fue llamado STOP (11) y posteriormente Essure.

El objetivo de esta comunicación es presentar los primeros cinco casos de esterilización permanente por vía histeroscópica con dispositivo intra tubario Essure.

\section{MATERIAL Y MÉTODO}

Entre enero y febrero de 2005, se realizaron 5 esterilizaciones de carácter permanente con dispositivo intra tubario (DIT) en pacientes del Departamento de Ginecología y Obstetricia de Clínica Las Condes. Estos cinco casos constituyen la marcha blanca del Programa de Contracepción Permanente.

Todas las pacientes firmaron un consentimiento informado una vez conocidos los detalles del procedimiento y sus posibles complicaciones. Se confeccionó una base de datos de registro prospectivo.

El rango de edad de las mujeres fue $37-42$ años y la paridad 2-4 hijos. Todas utilizaban previamente anticoncepción hormonal oral (Tabla I). A todas las pacientes se les realizó ecografía transvaginal en la semana previa, y se les administró 6 horas previas al procedimiento $200 \mu \mathrm{g}$ de misoprostol vía oral.

Para la esterilización se utilizó el sistema de contracepción Essure de Conceptus Inc., consistente en un micro espiral formado de fibras de poliéster (Polietileno terephtalato o Dacron) y metales (níquel, titanio y acero inoxidable). Este dispositivo tiene forma de espiral, expansible y con memoria dinámica una vez situado en la trompa. Mide $4 \mathrm{~cm}$ de longitud y $0,8 \mathrm{~mm}$ de grosor. Este dispositivo se encuentra acoplado a un cable de colocación y a un catéter de liberación. Todo este sistema a su vez está envainado dentro de un catéter que posee un mango para guiar la colocación y despliegue del DIT (Figura 1).

Técnica de inserción: El procedimiento se realizó en el pabellón de cirugía ambulatoria. Se utilizó en las tres primeras pacientes: midazolam 5 $\mathrm{mg}$, fentanilo $100 \mathrm{mg}$ y propofol $50 \mathrm{mg}$; en las dos restantes solo propofol $150 \mathrm{mg}$. Paciente en posición ginecológica. Aseptización vaginal con povidona yodada. Se utilizó un histeroscopio con óptica de 30 grados, camisa de $7,6 \mathrm{~mm}$ y canal operatorio de 5 french. Distención de la cavidad uterina con solución salina tibia $\left(37^{\circ} \mathrm{C}\right)$ mediante perfusión por gravedad. Inspección de la cavidad identificando ambos ostium, luego se sigue con la técnica quirúrgica específica (Figura 2).

Paso 1: Inserción del introductor en el canal de trabajo del histeroscopio. Se pone el sistema de colocación Essure a través del lumen del introductor y se avanza a través del canal del histeroscopio.

Paso 2: Avance del sistema de colocación en la trompa proximal con movimiento lento y sostenido para evitar espasmos. Se realiza hasta que la marca de posicionamiento esté en el ostium tubario (Figura 2).

Paso 3: Extracción del catéter de colocación. Se gira la rueda que trae el mango a ritmo de un clic por segundo hasta que no pueda girar más, facilitando la extracción del catéter de colocación. De esta manera queda expuesto el DIT acoplado al catéter de liberación.

Paso 4: Extracción del catéter de liberación. Para ello se oprime un botón que permite seguir girando la rueda que extrae el catéter de libera-

Tabla I

CARACTERÍSTICAS DE LAS PACIENTES SOMETIDAS A ESTERILIZACIÓN PERMANENTE HISTEROSCÓPICA Y LAS COMPLICACIONES INTRAOPERATORIAS

\begin{tabular}{lccccc}
\hline Caso & Edad & Paridad & $\begin{array}{c}\text { Anticoncepción } \\
\text { previa }\end{array}$ & $\begin{array}{c}\text { Inserción } \\
\text { (minutos) }\end{array}$ & $\begin{array}{c}\text { Complicaciones } \\
\text { intraoperatoria }\end{array}$ \\
\hline 1 & 39 & 4 & Oral & 25 & No \\
2 & 41 & 2 & Oral & 30 & No \\
3 & 42 & 2 & Oral & 12 & No \\
4 & 41 & 3 & Oral & 10 & No \\
5 & 37 & 2 & Oral & 10 & No \\
\hline
\end{tabular}




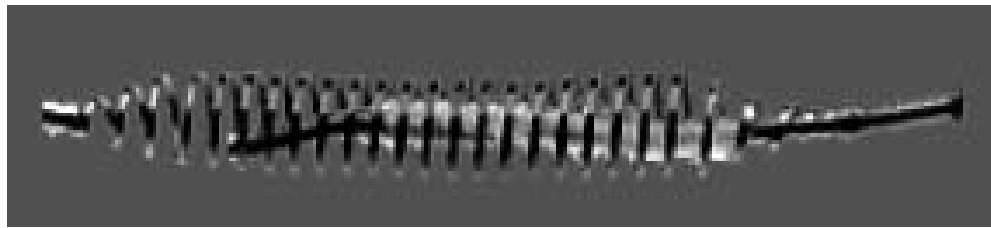

Figura 1. Dispositivo intra tubario Essure.

ción. Luego se puede observar como el DIT se expande. Se extrae el sistema de colocación a través del canal de trabajo del histeroscopio. La posición final del espiral debe ser con 3 a 8 vueltas fuera del ostium, hacia el útero (Figura 3).

Finalizado el procedimiento se mantuvo a la paciente en observación sólo por una hora, con alta hospitalaria para reintegrarse a las actividades habituales. Antes del alta se les preguntó por el grado de satisfacción (alta, media, insatisfecha) y tolerancia (muy buena, buena, mala) por el procedimiento. Además se les preguntó si recomendarían el procedimiento como opción para esterilización permanente y por qué razones. Se controló telefónicamente a las 24 horas y días 7, 15 y 30 post inserción.

\section{RESULTADOS}

Se logró colocar el dispositivo intra tubario de esterilización permanente en ambas trompas en los cinco casos. No hubo complicaciones intraoperatorias. No se observó espasmo ni perforación tubaria. El tiempo operatorio (desde entrada a

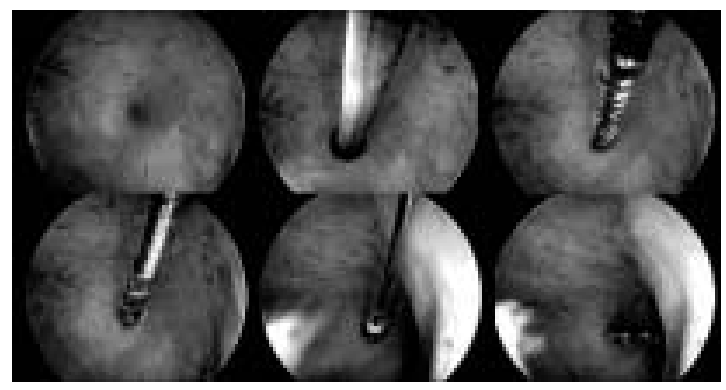

Figura 2. Procedimiento de inserción del DIT.

salida del histeroscopio) fluctuó entre 10 y $30 \mathrm{mi}-$ nutos. Los primeros dos casos 25 y 30 minutos. Los últimos tres casos 12, 10 y 10 minutos respectivamente.

Durante el período de observación postoperatorio todas expresaron haber tolerado muy bien el procedimiento de inserción y un grado de satisfacción alta. Además todas recomendarían el procedimiento por su rapidez, carácter ambulatorio y ausencia de dolor importante (Tabla II).

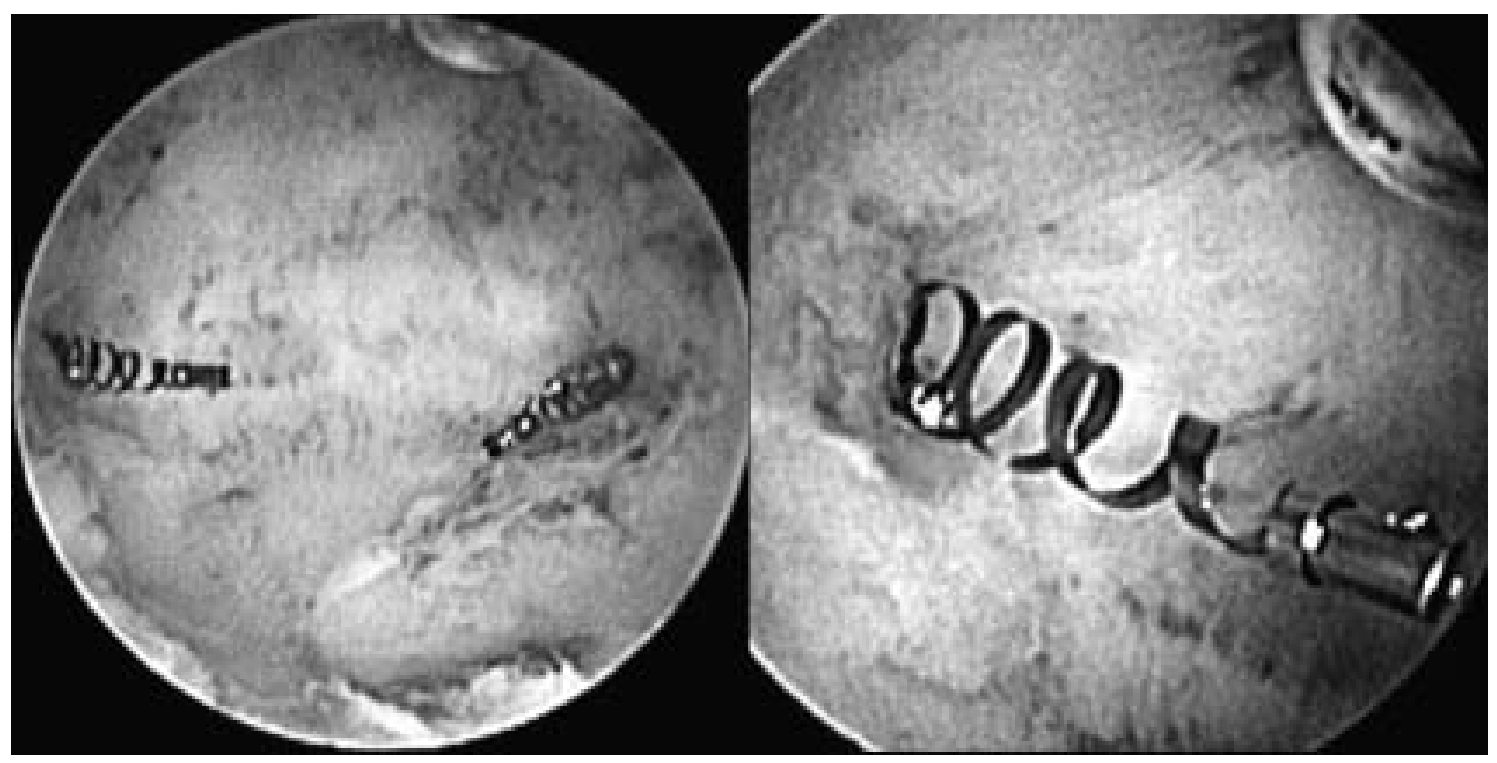

Figura 3. Posición final de los DIT. El espiral debe quedar con 3 a 8 vueltas fuera del ostium, hacia el útero. 
Tabla II

GRADO DE SATISFACCIÓN Y ACEPTACIÓN DEL PROCEDIMIENTO DE INSERCIÓN DEL DISPOSITIVO INTRA TUBARIO *

\begin{tabular}{lccc}
\hline Caso & $\begin{array}{c}\text { Grado de } \\
\text { satisfacción }\end{array}$ & Tolerancia & $\begin{array}{c}\text { Recomen- } \\
\text { dación }\end{array}$ \\
\hline 1 & alto & muy buena & sí \\
2 & alto & muy buena & sí \\
3 & alto & muy buena & sí \\
4 & alto & muy buena & sí \\
5 & alto & muy buena & sí \\
\hline
\end{tabular}

*Encuesta aplicada a las pacientes durante el período de observación (1 hora) previo al alta hospitalaria.

En el postoperatorio se registró un caso de metrorragia leve que se mantuvo por 18 días. Esta paciente además presentó dolor tipo menstrual por 19 días, moderado y con buena respuesta a analgésicos y antiinflamatorios vía oral (ácido mefenámico $500 \mathrm{mg}$ y luego ibuprofeno $200 \mathrm{mg}$ ). Se trató y controló ambulatoriamente (Tabla III).

\section{DISCUSIÓN}

Se trata de un método de anticoncepción permanente que tiene la gran ventaja de no requerir incisiones abdominales (12), como la esterilización por minilaparotomía o por vía laparoscópica. Además se puede realizar con sedación y analgesia. Todo esto disminuye la posibilidad de complicaciones derivadas de procedimientos quirúrgicos anestésicos. Es un método ambulatorio que permite una rápida reincorporación laboral, y que reduce por consiguiente los gastos de pabellón y medicamentos.

Este dispositivo al poseer una forma de espiral que se expande una vez posicionado en el interior de la trompa, queda ajustado a las paredes, evitando su expulsión. De esta manera se evita esta complicación que presentaban los otros métodos de esterilización oclusivos histeroscópicos (13). Logrando la obstrucción del lumen de la trompa por fibrosis.

Este método de esterilización permanente ha sido aprobado por la Comunidad Europea (febrero 2001) y por la FDA (noviembre 2002), debido a su demostrada seguridad y efectividad (11, 12, 14-18).

Diferentes publicaciones muestran éxito en el procedimiento de posicionar correctamente el DIT entre $85 \%$ (17) y $95 \%$ (12). Las fallas descritas se deben a obstrucciones por alteración de la estructura de la trompa, como consecuencia de infeccio-
Tabla III

COMPLICACIONES POSTOPERATORIO

\begin{tabular}{lll}
\hline Caso & Metrorragia (días) & Dolor (días) \\
\hline 1 & No $(-)$ & No \\
2 & No $(-)$ & No \\
3 & Leve (18) & Moderado (19) \\
4 & No $(-)$ & No $(-)$ \\
5 & No $(-)$ & No $(-)$ \\
\hline
\end{tabular}

nes en su mayoría. Sin embargo se describen casos de espasmo debido a la manipulación de la trompa. En nuestros casos se logró situar el DIT, sin presentarse esta complicación.

Las complicaciones intraoperatorias son muy escasas al tratarse de un procedimiento minimamente invasivo. Sin embargo debe tenerse en cuenta las derivadas de una técnica histeroscópica incorrecta. Por ello debe realizarse por ginecólogos con experiencia en histeroscopía.

Se han descrito complicaciones durante o inmediatamente después del procedimiento como náuseas y vómitos que son leves y pasajeros. Que pueden tratarse con medicamentos de ser necesarios, y que no se presentaron en nuestros casos. Igualmente se han descrito casos de metrorragia leve a moderada entre 1 a 15 días $(<1 \%)$, la cual se presentó por 18 días en un caso, sin necesidad de intervención mayor. El dolor tipo menstrual leve a moderado es otro síntoma que pueden presentar luego de la intervención, lo que también fue observado en la misma paciente de la metrorragia.

Es importante tener presente que se trata de un método de esterilización permanente, lo cual debe recalcarse a la paciente al momento de su decisión. Debe tratarse de pacientes con paridad deseada cumplida, ya que no se puede recuperar la fertilidad.

El espiral logra una obstrucción completa de la trompa a los tres meses, tiempo en el cual deberá realizarse una histerosalpingografía para corroborar que se ha logrado la obstrucción. Se ha descrito la ecografía como método para verificar la correcta posición del DIT (19).

A pesar que se trata de la comunicación de los resultados preliminares de sólo 5 pacientes, los primeros en el país, y sobretodo en base a las publicaciones existentes en Europa y Estados Unidos, podemos decir que se trata de un procedimiento de gran aceptación y satisfacción por parte de la paciente. 
Además es de gran seguridad al ser escasas las complicaciones, y gran eficacia para evitar el embarazo. No se ha informado de falla del método.

Esta comunicación ha revisado la tolerancia y aceptación del procedimiento de inserción histeroscópico del DIT y sus complicaciones. Continuaremos el seguimiento de estas pacientes y agregaremos nuevos casos, para analizar la efectividad del método a largo plazo.

\section{BIBLIOGRAFÍA}

1. Ross JA. Sterilization: past, present and future. Stud Fam Plann 1992; 23: 187-98.

2. Petterson HB, Xia Z, Wilcox LS, Tylor LR, Trussell $\mathrm{J}$, for the US Collaborative Review of Sterilization Working Group. Pregnancy after tubal sterilization with silicone rubber band and spring clip application. Obstet Gynecol 2001; 97: 205-10.

3. Scwartz DB, Wingo PA, Antarsh L, Smith JC. Female Sterilization in the United States 1987. Fam Plann Perspect 1989; 21: 209-12.

4. Mosher WD. Contraceptive practice in the United States, 1982-1988. Fam Plan Perspect 1990; 22 : 198-205.

5. Destefano F, Greenspan JR, Dicker RC, Peterson HB, Strauss LT, Rubin GL. Complications of interval laparoscopic tubal sterilization. Obstet Gynecol 1983; 61: 153-8.

6. Peterson HB, Destefano R, Rubin GL, Greenspan JR, Lee NC, Ory HW. Deaths attributable to tubal sterilization in the United States, 1977-1981. Am J Obstet Gynecol 1983; 146: 131-36.

7. Siegler A, Valle RF. Therapeutic hysteroscopic procedures. Fertil Steril 1988; 50-9.

8. Davis RH, Erb R, Kyriazis GA, Balin H. Fallopian tube occlusion in rabbits with silicone rubber. $J$ Reprod Med 1975; 14: 56-61.
9. Van De Leij G, Lammes FB. Office hysteroscopic tubal occlusion with siloxane intratubal devices (the Ovabloc method). Int J Gynaecol Obstet 1996; 53: 253-60.

10. Reed TP, Erb RA. Hysteroscopic occlusion with silicone rubber. Obstet Gynecol 1983; 61: 388-92.

11. Valle RF, Carignan CH S, Wrigt TC. Tissues response to the Stop microcoil transcervical permanent contraceptive device: results from prehysterectomy study. Fertil Steril 2001; 76: 974-80.

12. Ubeda A, Labastida R, Dexeus S. Essure: a new device for hysteroscopic tubal sterilization in an outpatient setting. Fertil Steril 2004; 82(1): 196-1999.

13. Kerin JF. New methods for transcervical cannulation of the Fallopian tube. Int J Gynecol Obstet 1995; 51: Suppl S29-39.

14. Kerin JF, Carignan CS, Cher D. The safety and effectiveness of a new hysteroscopic method of permanent birth control: results of the first Essure pbc clinical study. Aust N Z J Obstet Gynaecol 2001; 41: 364-70.

15. Lopes P, Menez C, Martigny H, Boudineau M. Sterilization for women using a hysteroscopic technique under local anesthesia. Press Med 2003; 32: 22-3.

16. Kerin JF, Cooper JM, Price T, Van Herendael B, Cayuela-Font E, Cher D, Carignan CS. Hysteroscopic sterilization using a micro-insert device: results of a multicentre phase II study. Hum Reprod 2003; 18: 1223-30.

17. Cooper JM, Carignan CH S, Cher D, Kerin JF. Microinsert nonincisional hysteroscopic sterilization. Obstet Gynecol 2003; 102: 59-67.

18. Cayuela E, Valle R, Cos R, Heredia F, Florensa R, Moros $\mathrm{S}$. Programa de adiestramiento y resultados en la inserción histeroscópica de dispositivos para la esterilización tubárica permanente. Prog Obstet Ginecol 2003; 46: 283-90.

19. Teoh M, Meagher S, Kovacs G. Ultrasound detection of the Essure permanent birth control device: a case series. Aust N Z J Obstet Gynaecol. 2003; 43(5): 378-80. 